\title{
An investigation into the effects of cognitive behavioral therapy on patients with chronic depression: a small case series
}

This article was published in the following Dove Press journal:

Psychology Research and Behavior Management

27 September 2012

Number of times this article has been viewed

\section{Gemma Louise Horn \\ University of Dundee, Scotland, UK}

Correspondence: Gemma Louise Horn Flat 6, 37 Waterfront Park, Edinburgh, Scotland EH5IEZ, UK

Tel +447970107865

Email gemmahorn@nhs.net
Background: National Institute for Clinical Excellence (NICE) guidelines recommend a combination of cognitive behavioral therapy (CBT) and antidepressants to treat chronic depression. The Cognitive Behavioral Analysis System of Psychotherapy (CBASP) is the only therapy model specifically designed for the treatment of chronic depression.

Objectives: To determine the clinical response to the CBASP of patients in a specialist clinical service for affective disorder and to ascertain their views on the value of the CBASP for their condition.

Methods: Qualitative data from interviews including a questionnaire and objective data from Becks Depression Inventory II symptom rating scales were used to monitor the progress of a small case series of five patients with chronic, treatment refractory depression as they received the CBASP over a 10-month period.

Results: Common themes from patient interviews show very positive engagement and attitudes to the CBASP from the questionnaire. Rating scales from Becks Depression Inventory II pre- and posttreatment showed very little change for three patients with improvements between 2 and 7 points but deterioration in symptoms of 2 points for the fourth patient.

Conclusion: The CBASP is a well-liked and positive therapy that helps patients manage their lives and deal with personal relationships, although objective data indicate little change in symptom severity.

Keywords: cognitive behavioral therapy, chronic depression, CBASP

\section{Background}

Depression is among the most common and serious of the mood disorders affecting people in the United Kingdom today. Approximately one in ten males and one in four females are affected by depression at some point in their lives. ${ }^{1}$ Depressed mood and a loss of interest or motivation are the key symptoms of depression, which is also characterized by low energy, poor concentration, a change in appetite and sleep pattern, weight loss, and feelings of worthlessness. ${ }^{1}$ Approximately two-thirds of depressed patients contemplate suicide and 10\%-15\% commit suicide. ${ }^{1}$

Depression is most frequently treated with antidepressants, but can also be treated with cognitive behavioral therapy (CBT) where it is available. ${ }^{2}$ CBT is a type of psychotherapy that helps a person change what they think and how they act. It aims at changing thinking patterns directly and focuses on present and future thinking. ${ }^{3}$ This type of therapy is not widely available to patients with depression. As depression can lead to an increased risk of suicide, this project will highlight why it is important that further treatment becomes more accessible to patients with chronic depression. 
Evidence suggests that a combination therapy of both antidepressant medication and CBT is effective in about two-thirds of patients with depressive episodes of mild to moderate severity. For a significant minority of patients $(20 \%-30 \%)$ who fail to respond to these therapies, the course of depression can be prolonged and very disabling. ${ }^{4}$ Chronic depression is defined by a person meeting the diagnostic criteria of the symptoms previously mentioned for depression for at least 2 years. ${ }^{5}$ National Institute for Clinical Excellence (NICE) guidelines recommend a combination of CBT and antidepressants for this type of depression. ${ }^{5}$ This recommendation is based on a recent clinical trial in the USA of a new form of CBT called the Cognitive Behavioral Analysis System of Psychotherapy (CBASP), which is currently being used in the Advanced Intervention Service at Ninewells Hospital, Dundee, UK. The research for this project was based in this center, which provides the CBASP to people with chronic depression using advanced psychological and medical interventions. This investigation will explore the value of the CBASP as a new form of CBT using data from patient interviews and the Beck Depression Inventory (BDI) II symptom rating scales in a small case series.

The CBASP is a form of CBT developed by Dr James McCullough. ${ }^{6}$ The CBASP model is interpersonal rather than intrapsychic or cognitive, and so the restructuring of cognitions plays only a minor role. The CBASP represents an approach that integrates mainly behavioral and interpersonal, but also cognitive and psychodynamic, strategies. This therapy uses structured exercises to help patients with depression redevelop their ability to determine the cause and effect of situations in interpersonal interactions, and subsequently change their thinking patterns. This is the only therapy model designed specifically for the treatment of chronic depression. ${ }^{6}$ Patients receiving the CBASP are also prescribed antidepressants. When receiving this therapy they are taught that their interpersonal behavior has certain consequences and, by learning to recognize these consequences, they gain insight and become reconnected to their environment. This is done using techniques and exercises like situational analysis, where a patient's perception and management of a situation is changed. The interpersonal discrimination exercise teaches patients to compare different examples of handling situations in their lives in relation to how they react to others in their lives. The final lesson is contingent personal responsivity, which helps stop the development of inappropriate behavioral thinking in situations that patients encounter. ${ }^{6}$
A literature review using "depression" and "CBT" search terms was performed on the PubMed database, with the articles viewed denoting different results about this form of treatment. Five articles reported positive evidence for CBT as a beneficial treatment in the management of chronic depression. ${ }^{4,7-10}$ One nonblinded, controlled trial used a treatment group and a control group. ${ }^{4}$ The treatment group received 12 sessions of CBT. Becks Depression Inventory readings were obtained from both groups and the treatment group showed a significant clinical improvement. A downside of this may involve the recall bias. Confounding factors between the treatment group and the control group were reduced as the two groups were matched by age, sex, and social support. ${ }^{4}$

One reason why patients do not receive CBT may be that some clinicians consider antidepressants to be superior. Five papers suggested that CBT was as good as antidepressants. ${ }^{11-15}$ Two trials have been undertaken to see the full benefits of CBT and the benefits of combination treatment. ${ }^{16,17} \mathrm{~A}$ doubleblind, placebo-controlled trial ${ }^{16}$ concluded that $\mathrm{CBT}$ and the antidepressant phenelzine are equally efficacious for improving symptoms of atypical depression. ${ }^{16}$ In that study, 142 patients were randomized into three groups according to the treatment modality, namely, CBT, phenelzine pharmacotherapy, and placebo.

Notwithstanding these promising results from a well-designed clinical trial, general practitioners may be reluctant to refer their patients for CBT due to potential cost considerations. Nonetheless, prior work suggests that $\mathrm{CBT}$ sessions are as cost-effective as prescribing antidepressants. ${ }^{7,11,18}$ One review ${ }^{7}$ took data from sources including pharmaceutical companies, psychiatrists, and the Australian health system, calculating the costs of drug treatment and CBT. The results showed that the price of a long-term course of selective serotonin reuptake inhibitor antidepressants exceeds that of the CBT sessions. This has led the Australian Government to improve the availability of CBT to depressed patients. ${ }^{18}$ An additional study showed how CBT could help reduce the number of patients with suicidal intent from committing suicide. ${ }^{19}$ As the risk of suicide in patients with depression is increased, it is important that CBT becomes more available to patients.

The clinical trials reviewed contained a mix of acute, atypical, and chronic depression. The evidence for the efficacy of CBT with chronic depression however was limited. This makes it difficult to know whether a treatment such as CBT is likely to be effective in a specialized clinic in a hospital setting (tertiary services), in which patients referred may be both treatment-refractory and chronic. This provides 
the rationale for looking at treatment response in the current investigation.

This project assessed the importance of CBT in the management of a small series of patients with chronic depression. The conclusion of this literature review suggests CBT is a potentially beneficial intervention for the management of chronic depression. In this case series I assessed how a small number of patients reacted to the CBASP method of CBT.

\section{Methods}

The purpose of this investigation was to conduct a small case series to examine how CBT affects patients with chronic depression using both qualitative data from interviews and objective data from symptom rating scales. I aimed to determine the patients' views on the value of receiving the CBASP for chronic depression and the proportion of patients with chronic depression receiving CBASP that made a clinically significant improvement. To enable me to discover patients' views about CBASP, I designed a questionnaire consisting of ten questions that concentrated on learning the patients' thoughts, knowledge, and perceived benefit of the therapy. Five patients with chronic depression were selected at random by a secretary, not involved in the project, from the CBASP outpatient therapy list. Due to this small number of patients, this study was designed as a small case series.

The inclusion and exclusion criteria for selected patients were the primary diagnosis of chronic major depression with no other major health comorbidities, including life-threatening conditions; patients were those who had experienced depression for over 5 years with a previous diagnosis of chronic depression made by a consultant psychiatrist; if a patient's medications or dose changed significantly during the investigation, they were excluded from the study. Minor dose adjustments in patients' medication were allowed. A letter was given to the patients informing them of the interview, timing, and that confidentiality of their personal details would be preserved. All included patients remained anonymous; no hospital numbers, names, or dates of birth were recorded. A consent form was also given to the patients and signed prior to the interview. The consent form and protocol were approved by the local Caldicott guardian, which overlooks ethical approval for research in this region. Interviews lasted 30 minutes and took place in the outpatient clinic. To gain information about what proportion of patients had improved after CBASP therapy sessions, Becks Depression Inventory (BDI) II scores were recorded at the start of therapy and just prior to the interview.

\section{Results}

\section{Interview results}

Most patients found the treatment helpful. It was described as interesting, practical, and valuable. It was better received than previous psychotherapy treatments as, according to the patients, it helped to prepare them for future situations and to deal with difficult issues. Patients developed objective reasoning and felt more confident about themselves. The treatment was rated highly as shown in Table 1 (see Appendix for more detail).

\section{BDI II analysis}

The BDI is a 21-question self-report inventory that is used for measuring the severity of depression. ${ }^{20}$ Each answer is scored on a scale value of 0 to 3 . The standard cut offs are as follows: 0-13 minimal depression, 14-19 mild depression, 20-28 moderate depression, and 29-63 severe depression. The BDI II results showed a varied pattern. Three patients showed a change of 2 to 3 points; the fourth showed a more significant increase of 7 points. The fifth patient had only two sessions of the CBASP, and therefore had only the initial score (see Tables 1 and 2).

\section{Discussion}

From the interview analysis it is clear that the patients considered the CBASP to be a positive experience. The five patients interviewed all provided similar responses to the questions asked. Unfortunately a BDI II posttreatment score was not available for Patient E. Their responses indicate they feel the therapy is helpful and has improved their overall mood. A common theme was that the patients found the sessions difficult, but the exercises they were given to perform at home were rated highly and were believed to be one of the key components of the CBASP. The patients all reported similar changes in their coping skills, including becoming more positive and able to deal with situations, more able to show emotions, and able to confront their own behavior. The average rating given for the therapy was 4.2 out of a maximum of 5, which indicates the therapy was well liked.

The BDI II results showed a varied pattern. Following analysis of the results, the majority of patients showed an increase in points, and therefore deterioration of their

Table I Patient treatment rating of the CBASP

\begin{tabular}{llllll}
\hline Patient & A & B & C & D & E \\
\hline Actual treatment rating $(I-5)$ & 4 & 4 & 5 & 5 & 3 \\
\hline
\end{tabular}

Abbreviation: CBASP, Cognitive Behavioral Analysis System of Psychotherapy. 
Table 2 Patient BDI II before and after the CBASP

\begin{tabular}{llllll}
\hline Patient & A & B & C & D & E \\
\hline BDI II before the CBASP & 5 I & 32 & 44 & 38 & 30 \\
BDI II after the CBASP & 53 & 30 & 47 & 45 & Not available \\
BDI II change & 2 & -2 & 3 & 7 & ??? \\
\hline
\end{tabular}

Abbreviations: BDI, Beck Depression Inventory; CBASP, Cognitive Behavioral Analysis System of Psychotherapy.

depressive symptoms. This discrepancy between the interview results, showing very positive engagement and attitudes to the therapy, and the BDI II results, show that the lack of change in symptom severity could be due to a number of factors. The therapy may improve a patient's ability to manage their lives and personal relationships, but may not change the disorder itself. Another possibility is that patients receiving the CBASP are significantly more severe and/or complex than those included in previous clinical trials, indicating the benefits of CBT. This is portrayed in the patient profiles. These patients have suffered for many years with severe depression and may need a more intensive treatment including combination of $\mathrm{CBT}$ and antidepressant medications or various augmentation strategies, such as repetitive transcranial magnetic stimulation and electroconvulsive therapy. Another possibility is that they may need a longer course of CBASP. A final point that may explain why the patients' symptoms did not improve is the trainee status of the therapist. Treatment by a more experienced provider could have produced different outcomes. As the interview results depicted optimistic information but the BDI II scores showed no improvement and, for some, deterioration of symptoms, it is possible that the CBASP may not be effective for this particular group of patients seen in the specialized service for chronic and treatment refractory affective disorder. However, the small collection of subjects included in the present report limits the interpretability of the findings.

When referring back to previous trials, it is clear that many have been successful in proving that CBT is a beneficial treatment. One trial, ${ }^{21}$ which used a similar method to that of this study, proved that BDI II scores from patients receiving CBT showed a clinical improvement. This trial used a larger number of patients (38) who all received the same number of sessions of CBT (12). Two trials that established the CBASP to be successful were also carried out using a larger number of patients. ${ }^{21,22}$ One trial assessed 850 patients over a 4-year period. This trial obtained positive results and was therefore used to establish the CBASP in different centers around the world. ${ }^{21}$ However, the patients in this trial suffered from chronic depression but not necessarily treatment-resistant depression; that is, a large number had not received adequate treatment in the past.

\section{Conclusion}

The results of this project show that CBASP is a positively received therapy that helps patients to manage their lives and to deal with personal relationships. The BDI II scores show that there was no improvement and, for some, a deterioration in symptom severity. Therefore, from the results of this small case series, the CBASP was not proven to improve symptoms of depression in this particular group of patients. Depression can be considered to have two components: the psychological or cognitive component, for example, mood, and the physical or somatic component such as loss of appetite. The BDI II reflects this and can be separated into two subscales. Hence, future analysis could be performed on the effects of the CBASP on both the cognitive and somatic aspects of chronic depression. ${ }^{23,24}$

Depression is a very common problem and a serious disability in society. Therefore, it is important that CBT and the CBASP are made more widely available to treat patients with depression. The percentage of patients that commit suicide each year further emphasizes this point. ${ }^{1}$ Previous research has confirmed that CBT can decrease the risk of suicide, ${ }^{19}$ a previous meta-analysis also showing that the combination of pharmacological and psychological therapies is an effective treatment for depression. ${ }^{25,26}$ From the patient interview answers, it is clear that the CBASP helps them deal with personal relationships and manage their lives, and makes them feel better.

\section{Acknowledgments}

I am grateful for the support given by Dr Robert Durham in all stages of this study. He provided me with design, procedural, and ethical guidance and presented my case to the NHS Tayside COREC committee to gain authority to interview psychiatric patients. He also assisted in gaining access to patients and patient medical records. I would also like to give thanks to the administration staff (Frances Armstrong) in Ninewells Hospital Advanced Intervention Service for allowing me to use their interview facilities, and to Professor Keith Matthews for providing access to patients and case records.

\section{Disclosure}

The author reports no conflicts of interest in this work. 


\section{References}

1. Masih S, Devilly G. Psychoeducation material on depression. Transition Counseling Services; 2008. Available from: http://cindylellis.wordpress. com/2008/07/07/psychoeducation-material-on-depression/. Accessed January 25, 2007.

2. Rcpsych.ac.uk [homepage on the Internet]. Antidepressants. London: Royal College of Psychiatrists; 2006 [updated Jun 2012]. Available from: http://www.rcpsych.ac.uk/mentalhealthinformation/ mentalhealthproblems/depression/antidepressants.aspx. Accessed January 25, 2007.

3. rcpsych.ac.uk [homepage on the Internet]. Cognitive Behavioural Therapy. London: Royal College of Psychiatrists; 2006 [updated Jan 2012]. Available from: http://www.rcpsych.ac.uk/mentalhealthinformation/therapies/cognitivebehaviouraltherapy.aspx. Accessed January 25, 2007.

4. Embling S. The effectiveness of cognitive behavioural therapy in depression. Nurs Stand. 2002;17(14-15):33-41.

5. National Institute for Clinical Excellence. Depression: The Treatment and Management of Depression in Adults. National Collaborating Centre for Mental Health; 2009. Available from: http://www.nice.org.uk/ nicemedia/pdf/CG90NICEguideline.pdf. Accessed January 25, 2009.

6. cbasp.org [homepage on the Internet]. Psychopathology and treatment. Richmond, VA: CBASP.org. Available from: http://www.cbasp.org/ psy.htm. Accessed January 24, 2008.

7. Lamers F, Jonkers CC, Bosma H, Diederiks JP, van Eijk JT. Effectiveness and cost-effectiveness of a minimal psychological intervention to reduce non-severe depression in chronically ill elderly patients: the design of a randomized controlled trial [ISRCTN92331982]. BMC Public Health. 2006;6:161.

8. Birmaher B, Brent DA, Kolko D, et al. Clinical outcome after short-term psychotherapy for adolescents with a major depressive disorder. Arch Gen Psychiatry. 2000;57(1):29-36.

9. Finucane A, Mercer SW. An exploratory mixed methods study of the acceptability and effectiveness of Mindfulness-Based Cognitive Therapy for patients with active depression and anxiety in primary care. BMC Psychiatry. 2006;6:14.

10. Oei TP, Bullbeck K, Campbell JM. Cognitive change process during group cognitive behaviour therapy for depression. JAffect Disord. 2006; 92(2-3):231-241.

11. Revicki DA, Siddique J, Frank L, et al. Cost-effectiveness of evidencebased pharmacotherapy or cognitive behavior therapy compared with community referral for major depression in predominantly low-income minority women. Arch Gen Psychiatry. 2005;62(8):868-875.

12. Jarrett RB, Schaffer M, McIntire D, Witt-Browder A, Kraft D, Risser RC. Treatment of atypical depression with cognitive therapy or phenelzine: a double-blind, placebo-controlled trial. Arch Gen Psychiatry. 1999; 56(5):431-437.
13. Levitan RD. Treatment of atypical depression with cognitive therapy or phenelzine. Arch Gen Psychiatry. 2000;57(11):1084-1085.

14. DeRubeis Rj, Hollon SD, Amsterdam JD, et al. Cognitive therapy vs medications in the treatment of moderate to severe depression. Arch Gen Psychiatry. 2005;62(4):409-416.

15. Schatzberg AF, Rush AJ, Arnow BA, et al. Chronic depression: medication (nefazodone) or psychotherapy (CBASP) is effective when the other is not. Arch Gen Psychiatry. 2005;62(5):513-520.

16. Churchill R, Wessely S, Lewis G. Combinations of Pharmacotherapy and Psychotherapy for Depression (Protocol). The Cochrane Library. John Wiley and Sons, Ltd; 2005.

17. Churchill R, Wessely S, Lewis G. Antidepressants alone versus psychotherapy alone for depression [Protocol for a Cochrane Review]. In: The Cochrane Library, Issue 4, 2003. Oxford: Update Software.

18. Vos T, Corry J, Haby MM, Carter R, Andrews G. Cost-effectiveness of cognitive-behavioural therapy and drug interventions for major depression. Aust N Z J Psychiatry. 2005;39(8):683-692.

19. Maris RW. Suicide. Lancet. 2002;360(9329):319-326.

20. Beck AT, Steer RA, Ball R, Ranieri WF. Comparison of Beck Depression Inventories-IA and -II in psychiatric outpatients. $J$ Pers Asses. 1996;67(3):588-597.

21. Keller MB, McCulloch JP, Klein DN, et al. Comparison of nefazodone, the cognitive behavioral-analysis system of psychotherapy, and their combination for the treatment of chronic depression. The N Engl J Med. 2000;342(20):1462-1470.

22. McCullough JP Jr. Treatment for Chronic Depression: Cognitive Behavioural Analysis System of Psychotherapy (CBASP). 1st ed. New York: Guilford Press; 2003.

23. Steer RA, Ball R, Ranieri WF, Beck AT. Dimensions of the Beck Depression Inventory-II in clinically depressed outpatients. J Clin Psychol. 1999;55(1):117-128.

24. Storch EA, Roberti JW, Roth DA. Factor structure, concurrent validity, and internal consistency of the Beck Depression InventorySecond Edition in a sample of college students. Depress Anxiety 2001; 19(3):187-189.

25. Cuijpers P, van Straten A, Schuurmans J, van Oppen P, Hollon SD, Andersson G. Psychotherapy for chronic major depression and dysthymia: a meta-analysis. Clin Psychol Rev. 2009;30(1):51-62.

26. Rupke S, Blecke D, Renfrow M. Cognitive Therapy for Depression. Am Fam Physician. 2006;73(1):83-86. 


\section{Appendix}

\section{Interview results}

\section{What did you think of the treatment?}

Patient A: I found the treatment to be positive and helpful. Patient B: I think it is starting to make a difference. My mood swings up and down, but that is a good change as it is usually down.

Patient C: I think it is very helpful but it took me a few months to get the hang of it.

Patient D: I think it is better than normal CBT as it goes into more depth.

Patient E: I think it is interesting and practical. It is different to CBT I have had in the past. It helps me structure incidents and analyze them.

\section{What did your therapy involve?}

Patient A: I have CBASP sessions once a week with something to accomplish for every session. I have exercises to do at home.

Patient B: I have sheets to take home to help me work through situations. It helps me work through things in slices and encourages me to speak out more.

Patient C: The aim of the therapy is to prepare me for situations and how to deal with people.

Patient D: It involves talking about family history and training me to think differently. It is quite helpful to be able to put things on paper.

Patient E: It involves me talking to a therapist about situations and people that have stamped on me.

\section{How valuable were the sessions?}

Patient A: I find them very valuable. They are an outlet for talking and improvement of my mood.

Patient B: I wasn't sure at the start but now I find them valuable.

Patient C: I find it very valuable. People don't hurt me like they used to and I can handle my family better.

Patient D: Yes, I find them very helpful but I think it depends on the person you are speaking to.

Patient E: They are valuable as they help me talk about things.

\section{Have you made an improvement? How?}

Patient A: Yes, I have developed more objective reasoning. It has given me hope and I feel like a burden has been lifted off my shoulders. The improvement has been slower than what I had hoped for though.

Patient B: Yes, I am less intimidated by people now. I am frightened I will not be able to hold onto this improvement though.
Patient C: Yes, I don't feel intimidated anymore and I have my own mind.

Patient D: Yes, a little bit. I am going to stick with it though.

Patient E: No, not yet as I have only had 2 sessions. Hopefully I will in the future though.

\section{How difficult were the sessions?}

Patient A: I find the session quite difficult and some situations I have to talk about are embarrassing. The written exercises were difficult at the beginning but now I find them easier.

Patient B: The sessions are quite difficult, a lot of concentration is needed.

Patient C: The sessions were hard to begin with as I was unsure what to do. Now they are ok.

Patient D: The written exercises are ok but the sessions are hard, you have to think a lot to find the answers.

Patient E: I found the sessions difficult. I became upset in the first session but felt stronger in the second.

\section{What changes would you make?}

Patient A: I would make no changes.

Patient B: I would make no changes.

Patient C: I would make no changes.

Patient D: I would make no changes.

Patient E: I would make no changes.

\section{How would you describe the therapy to others?}

Patient A: It allows you to confront your behavior and think things through. It also helps me to look into the future with hope.

Patient B: I would say it works well on chronic depression but this may not be the same for one episode of depression. You have to be able to concentrate fully.

Patient C: I would tell people the therapy is helpful and explain the way it has helped me. I think courage is needed to start and continue with the therapy.

Patient D: I would advise other people to take it. I would tell them how it is different to other therapies.

Patient E: I would tell them that it helps you to focus on thoughts and emotions that have become strong from the depression.

\section{What have you learned?}

Patient A: I have learned not to overthink situations and be negative. I have also learned that I do not need to fit in.

Patient B: I have learned lots about myself. I always had a mask on before but now that is coming off and I am learning to show emotions. 
Patient C: I have learned how to stand up for myself and how to be more assertive.

Patient D: I have learned not to assume things about situations and look at the whole picture first before I make a judgment. I can also look into the past better and with a new light.

Patient E: I have not learned much about myself yet but I have learned how the therapy works.

\section{What treatment do you prefer?}

Patient A: I prefer therapy as tablets do not change much and I don't want to be on tablets forever.

Patient B: Both, I need the medication to be able to cope with the therapy.
Patient C: I like both medication and therapy together. Patient D: I like both medication and therapy together.

Patient E: I liked psychotherapy and I found CBT quite helpful.

10. How would you rate the treatment on a scale of 1 to 5 where 5 is the highest rating?

Patient A: 4.

Patient B: 4 .

Patient C: 5 .

Patient D: 5 .

Patient E: 3.

Psychology Research and Behavior Management

\section{Publish your work in this journal}

Psychology Research and Behavior Management is an international, peerreviewed, open access journal focusing on the science of psychology and its application in behavior management to develop improved outcomes in the clinical, educational, sports and business arenas. Specific topics covered include: Neuroscience, memory \& decision making; Behavior

modification \& management; Clinical applications; Business \& sports performance management; Social and developmental studies; Animal studies. The manuscript management system is completely online and includes a quick and fair peer-review system. Visit http://www.dovepress. com/testimonials.php to read real quotes from published authors. 